\title{
OBSERVATION OF THE SPECTRUM OF COMET BORRELLY MADE WITH THE THIRTY-SIX INCH REFRACTOR
}

The spectrum of the Comet Borrelly was observed on July 14 and 15 with Spectrograph I (ef. L. O. Bulletin, No. 8). Visually it showed a relatively strong continuous spectrum and the three characteristic bands. Of these that at $\lambda 4700$ seemed the brightest, while the other two were much fainter.
The attempt was made to secure a photograph of the spectrum with an exposure of six hours, using the same instrument, but the intrinsic brightness of the comet is quite small, and only the merest trace shows on the plate in the region near $\lambda 4700$.

July $17,1903$.

\section{H. D. CurTis.}

\section{PRELIMINARY NOTE ON PHOTOGRAPHIC OBSERVATIONS OF COMET $c, 1903$ (BORRELLY)}

Photographic observations of Comet $c, 1903$ (Borrelly), were begun at the Lick Observatory on the night succeeding that of discovery, and are still in progress. Nine exposures ranging in length from thirty-eight minutes to four hours have been made with the Dallmeyer camera of $15 \mathrm{~cm}$. aperture, 82.6 $\mathrm{cm}$. equivalent focal length, and $14^{\circ} \times 16^{\circ}$ angular field. Three negatives with the same range of exposure have been obtained simultaneously with the Floyd camera of $13 \mathrm{~cm}$. aperture and $178 \mathrm{~cm}$. focal length.

Two tails, one of which is nearly straight, the other greatly curved, appear on all but the last plate. The curved tail is short and very bright while the straighter tail is relatively fainter and longer but developes some very interesting forms.

On a plate of four hours' exposure on June 23d, the tails are widely separated. The strait tail is narrow at the head, but broadens and extends $1 \frac{1}{2}$ degrees. On the plate of June 29th the longer tail has split up into two distinct branches which emerge separately from the head and diverge slightly as they extend into space. With four hours exposure on June 30th the secondary tail appears very bright with a length of $1 \frac{1}{2}$ degrees. The primary extends five degrees from the nucleus. The form is indistinct, but the division of the previous night still persists. One branch remains straight while the other is broader and longer and follows a sinuous curve. On the following night the strait tail is sharp and single.

The plates of July 12th and 13th still show two tails. The primary tail is straight and narrow and the secondary tail still greatly curved. On the plate of July 12th, with $38 \mathrm{~min}$. exposure, the primary tail is four degrees long; the secondary tail is 1.5 degrees in length. The plate of July 14th, $1 \mathrm{hr}$. $48 \mathrm{~min}$. exposure, shows one straight narrow tail, $8 \frac{1}{2}$ degrees in length, widening out slightly as it extends away from the head.

On all the plates the nucleus is sharp and approximately central with respect to the heavy coma.

During these exposures the earth has been approaching rapidly to the plane of the comet's orbit. At the same time the apparent angle between the tails has steadily diminished until, on the plate of July 14th, they are coincident. Measures of the position angles of the tails on two plates are given below. $P_{a}, P_{b}$ and $P_{r}$ represent respectively the position angles of the primary tail, secondary tail, and radius vector from the sun to the comet. The difference $P_{a}-P_{r}$ appears in the last column.

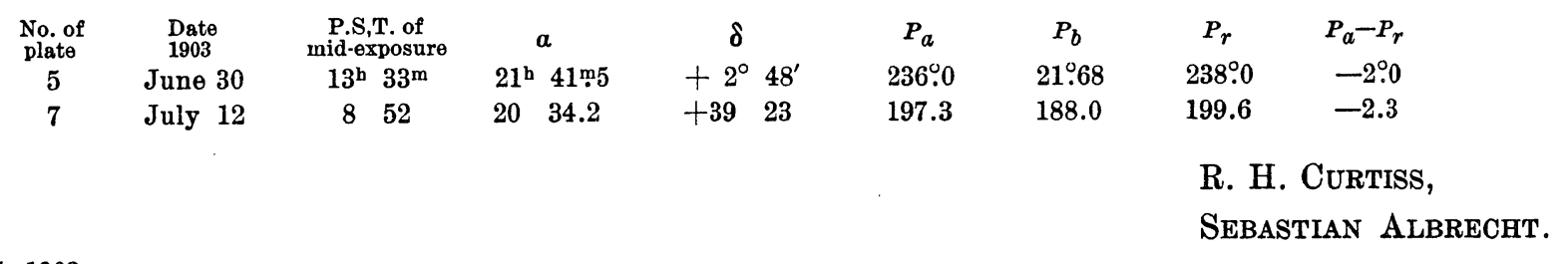

July 15, 1903. 